\title{
A Scalable Large Format Display Based on Zero Client Processor
}

\author{
Sang Don Kim, Seung Eun Lee \\ Department of Electronic Engineering, Seoul National University of Science and Technology, Republic of Korea
}

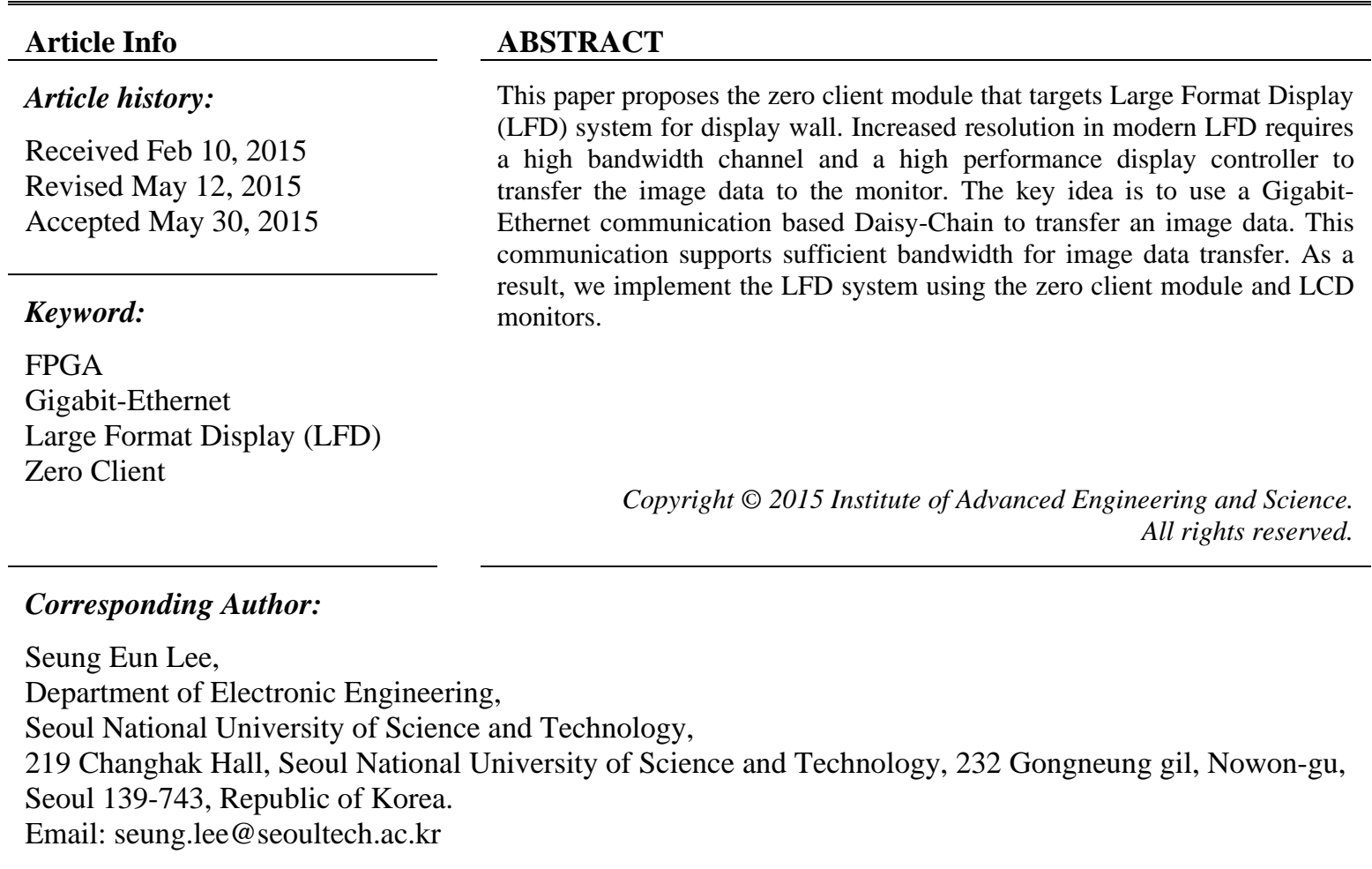

\section{INTRODUCTION}

The size of single LCD screen has continuously increased accodring to the development of semiconductor process. The larger LCD screen requires the Large Format Display (LFD) which connects the multiple LCD screens to implement a large display for an advertisement such as billboards. The LFD system requires the high performance display controller to drive the multiple screens. However, this is very expensive because the display controller includes the high performance graphic card for displaying via HDMI or VGA interface. These traditional interfaces have been restricted to transmission distance in dozen of meters. In case of large display over maximum transmission distance, the repeater should be adopted, and it leads to increase of the cost for the LFD system. In order to substitute the traditional display cable, a Gigabit-Ethernet can be adopted. The Gigabit-Ethernet supports longer distance to the data transmission, and it has sufficient bandwidth for the LFD.

The LFD system has been studied using various architecture. Rudolfs et al. proposed video wall controlled by mini PC [1]. Kim et al. proposed the controlling method for the video wall based on the network [2]. A central control has simple architecture, and it does not use a separate controller for each display. On the independent control, each display module controls the video signal provided from the supply unit. Subash et al. proposed the Gigabit-Ethernet based data acquisition system for imaging array [3]. High resolution image for a single phothon X-ray system was successfully transmitted through Gigabit-Ehternet [4].

In this paper, we propose the scalable LFD architecture using the client system which includes the Gigabit-Ethernet interface. The rest of our paper is organized as follows. Section 2 describes about the background of client systems and LFD. And we briefly introduces the features of the LFD system and the 
zero client modules in section 3, and present the hardware implementation of our prototype in Section 4. Section 5 shows the experimental result and Section 6 concludes this paper by outlining the direction for future work on this topic.

\section{CLIENT SYSTEMS AND LFD}

The Gigabit-Ethernet based screen image transmission have been used on the client systems. The client system, which is a kind of the remote access terminal, receives image data to control LCD monitor through the Gigabit-Ethernet and transmits the user's input such as keyboard or mouse. In order to support personal desktop environment, Virtual Desktop Infrastructure (VDI) allows to gain access to the desktop environment through the Gigabit-Ethernet communication. It supports single or multiple screen output for convenience. The client system depends on a server to support high performance computation. This client system is divided into three categories: a fat client, a thin client and a zero client. Among these systems, the fat client has a high performance processor and operating system. Even though this client system provides rich functionality and it can process many programs in a multitasking environment, it consumes much of power. On the other hand, The thin client consists of the low performance processor and operating system. It has no sufficient performance for program which requires high computation power. Because the server supports sufficient processing power to execute most of a user's program, the performance of the client system is not significant. Also, It has a low price and low power properties. However, the thin client consumes much of power than the zero client system. The zero client has the simplest hardware which is optimized for a remote client system. On the zero client, the operating system is not essential because the zero client system establishes the VDI channel using the Gigabit-Ethernet to support the personal desktop environment. A zero client system depends on the server to support computation power and hardware resources, and it implement the similar user interface based on the computational power of the server. Most of the program execution is processed on the server, and the client receives the processing result as a screen image. In terms of power consumption, the zero client is proper to the LFD system because the fat client and the thin client spent much of power than the zero client.

The Gigabit-Ethernet based LFD system is similar to zero client system because the screen image is fed to LCD screen through Gigabit-Ethernet. However, the Gigabit-Ethernet transmission suffers the bottleneck when it drives multiple LCD screens on the LFD system. Therefore, the image compression algorithm is used on the VDI. In order to avoid the bottleneck on the Gigabit-Ethernet channel, we adopt the Daisy-chain connection. The Daisy-chain is a wiring scheme in which multiple devices are connected together in series. It reduces the risk of a bottleneck on the Gigabit-Etnerhet channel because each serial ethernet connection establishes the local network. This Daisy-chain based on the Gigabit-Ethernet can be used for the LFD system because LFD system consists of the multiple LCD screen and the client system which is controls the screen. Vendors have developed a zero-client based display, which support streamline business IT environment with VDI. The thin client can be used for the secure VDI environment [5]. In order to establish secure VDI, the PC-over-IP (PcoIP) processor based zero client system [6] and the zero client using the PCoIP chipset, supporting VMware [7] were developed. The cloud connect is similar with these client systems and it has portability [8]. An architecture for zero client based LFD system was proposed [9].

In our system, each LCD screen of the LFD system has a single zero client module and each module is combined with Daisy-chain based on the Gigabit-Ethernet. The proposed LFD system has scalable properties thanks to the feature that the LCD screen can be attached or detached. And this is controlled by the host computer such as personal computer via Gigabit-Ethernet.

\section{ZERO CLIENT MODULE FOR SCALABLE LFD SYSTEM}

Most existing LFD consists of a display part and a display controller (See Figure 1(a)). The display part includes multiple LCD monitors, and the display controller supports numerous monitor output ports in order to control the display part. Therefore, it requires a high computation power for video processing. To reduce the performance requirement, we adopted the Daisy-Chain connection to our proposed LFD system (See Figure 1 (b)). An image display controller, which is on the display controller part, splits the image for display part, and transmits the divided image fragment with an ID. On the display part, a single zero client module controls a display monitor, and each of them is connected in Daisy-Chain using the Gigabit-Ethernet. Each zero client module has a unique module ID on the LFD system, and it enables classification of each monitor. The image data can be displayed when the received fragment ID is matched with the module ID of zero client module. Otherwise, received data is delivered to the next module through Daisy-Chain. Therefore, the image display controller does not require high performance, and Gigabit-Ethernet connection supports sufficient communication bandwidth for LFD system. 


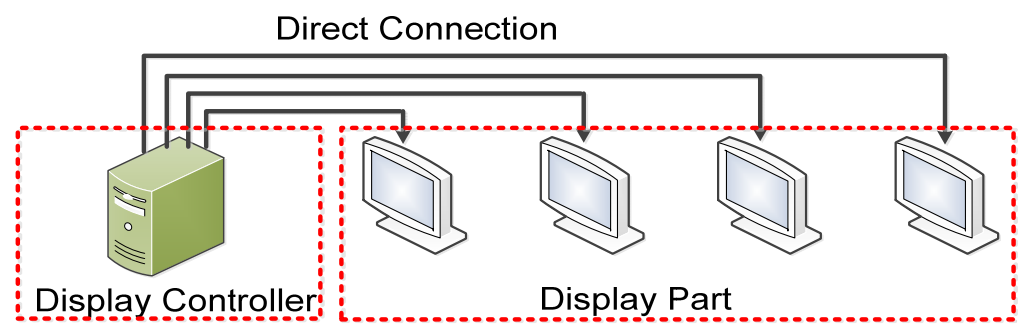

(a)

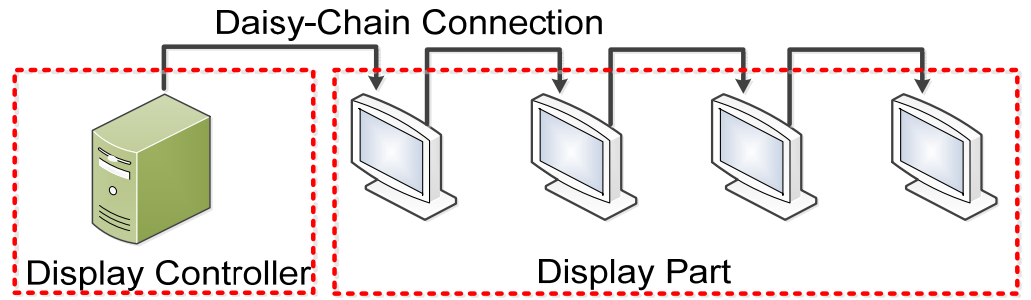

(b)

Figure 1. Zero Client Module for Scalable Lfd System; (a). Structure of the existing LFD system,

(b). Structure of the proposed LFD system

\subsection{Little Core for Zero Client System}

The Nios II core is a soft core which is synthesizable for FPGA. This core is optimized for performance according to various configurations in the LFD system. One of the types of Nios II core in accordance with configurations is a Nios II/s core which is a standard core to remove a significant trade-off in software performance. This core supports the ethernet IP for Altera FPGA and has sufficient performance to manage the flow of image data. We establish two Gigabit-Ethernet channel on the FPGA because of the Daisy-Chain requires two communication channels.

In order to process a large number of the image data, available memory space should be large to store image data in memory. We adopt the SDRAM as the system memory that supports sufficient memory space for maganging the image data. The SDRAM memory controller can be generated by means of Qsys tool and is exploited to utilize the SDRAM in our system. However, clock synchronization problems according to the I/O timing of FPGA occur between the SDRAM controller and the external SDRAM (See Figure 2). To resolve this problem, the SDRAM controller and the Nios II/s core operate with different clock, which has a faster phase about 1.5ns than that of the external SDRAM, generated by the PLL.

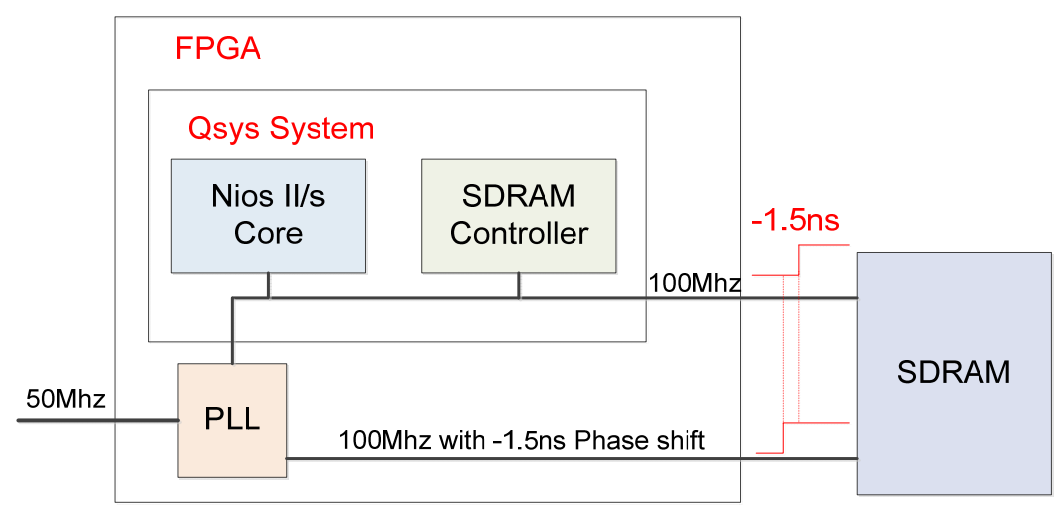

Figure 2. Block diagram of SDRAM controller

\subsection{Gigabit-Ethernet for Daisy-Chain}

The Triple Speed Ethernet MAC (TSE MAC) IP is provided for developer who designs hardware with the ethernet communication, and and it supports $10 \mathrm{Mbps}, 100 \mathrm{Mbps}$ and $1 \mathrm{Gbps}$ speed on ethernet connection. In our LFD system, the auto-negotiation function of Gigabit-Ethernet selects operation speed 
among the three ethernet speeds. The Nios II/s core controls the operation of sub blocks such as the TSE MAC core, the Scatter-Gather Direct Memory Access (SGDMA) TX, and the SGDMA RX by setting registers of each IP (See Figure 3). To support high-speed data transfer between a memory and the TSE MAC core, the SGDMA transfers data from system bus interconnect to streaming interface, and vice versa. In our system, two SGDMAs, which implement the role of transmitter and receiver, are connected to the TSE MAC core on the side of the streaming interface to eliminate congestion of system bus caused by massive data on the ethernet communication. The descriptor memory includes a series of descriptors, which involve information about the data to be transferred. Because the output clock generated from the oscillator of the FPGA is not an ideal signal, the DDIO (Double Data rate Input Output) module is used for creating accurate edge-aligned transmission clock of the external PHY chip.

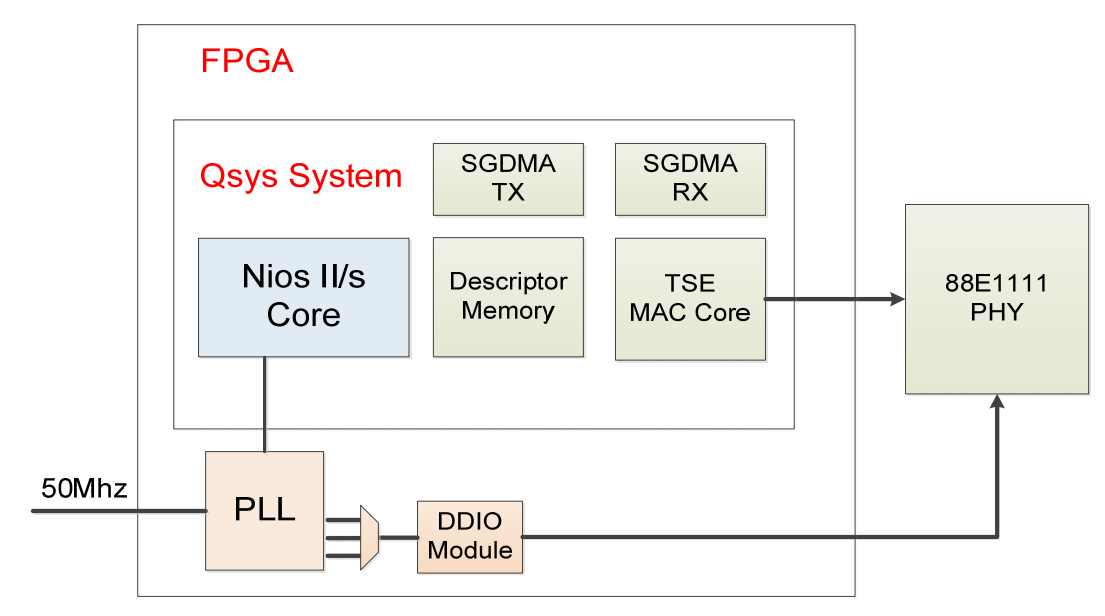

Figure 3. Block diagram of Triple Speed Ethernet (TSE) hardware

\subsection{VGA Controller}

The VGA controller visualizes the received data to the monitor. The resolution of our prototype is 640 x 480 and it supports 24 bit true color. The VGA controller has an image buffer that stores image frame to display. We adopt the SRAM buffer to optimize the system performance, and it enables fast image display. Figure 4 illustrates the block diagram of the VGA controller.

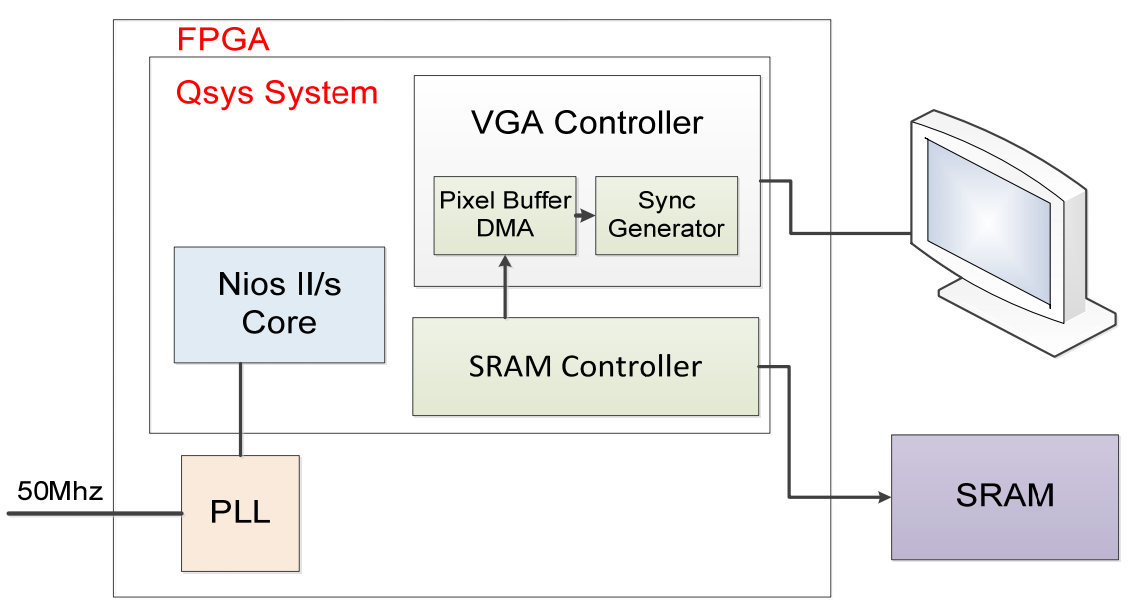

Figure 4. Block diagram of VGA controller

Because the monitor display operation has the highest priority in this block, pixel buffer DMA reads data from the SRAM as soon as possible. The Nios II/s core stores an image data to the SRAM when the memory is available. 


\section{IMPLEMENTATION}

We adopt the Altera DE2-115 board [10] for implementation of the zero client module and the proposed LFD system whose features include Altera Cyclone IV FPGA, 128MB SDRAM, 4MB SRAM, and two Ethernet PHY chips. Our system includes a Nios II/s core, an Avalon system bus, an SDRAM memory controller, a two Gigabit-Ethernet interfaces, a VGA controller and a JTAG debugging hardware. Nios II/s core is provided by Altera, and it supports 2 GB of external memory address space. The SDRAM controller is controlled by the core, and the $128 \mathrm{MB}$ of SDRAM is available. Two Gigabit-Ethernet is used for DaisyChain connection with other modules. It provides the sufficient bandwidth for image data transmission. The VGA controller integrates an SRAM controller that used for an image buffer.

In order to construct the LFD system using a zero client module, communication packet should be configured for image data transmission. In the our implementation, we use the UDP protocol because of its simplicity. This protocol has a small packet header compare to the TCP/IP protocol. Although this packet does not support bidirectional error correction algorithm, it is not critical in this system.

\section{EXPERIMENTAL RESULTS}

In order to verify the whole system, one zero client module is used for the image display controller. This module operates at $100 \mathrm{MHz}$ and it process the image data for the LFD system. In our verification environment, this zero client module reads the $640 \times 480$ of image data from the file system of the host computer and splits image on each fragment to construct a UDP packet. The UDP packet, which contains the image fragment and the necessary protocol headers, is transmitted to the display part through GigabitEthernet.

The display part consists of the two zero client modules and the LCD monitors. Each module contains the $128 \mathrm{MB}$ of SDRAM and the $4 \mathrm{MB}$ of SRAM, and these operate at $100 \mathrm{MHz}$. The SRAM is used for the image display buffer and it supports up to $1024 \times 768$ resolution. On the display part, one of the zero client module has module ID 0, and another one has a module ID 1 (See Figure 5). The received UDP packet contains a part of the display image, and the part of the image is 20 pixels in our prototype. The zero client module displays the received image data through Daisy-Chain when the fragment ID matches with module ID. Otherwise, the image data pass to the next module using Daisy-Chain through the Gigabit-Ethernet. Finally, the $60 \mathrm{~Hz}$ of $640 \times 480$ resolution of the image has been printed on the two LCD screens; the extended display resolution is $1280 \times 480$. In addition, the display part can be extended to multiple monitors because the connection structure has expandability.

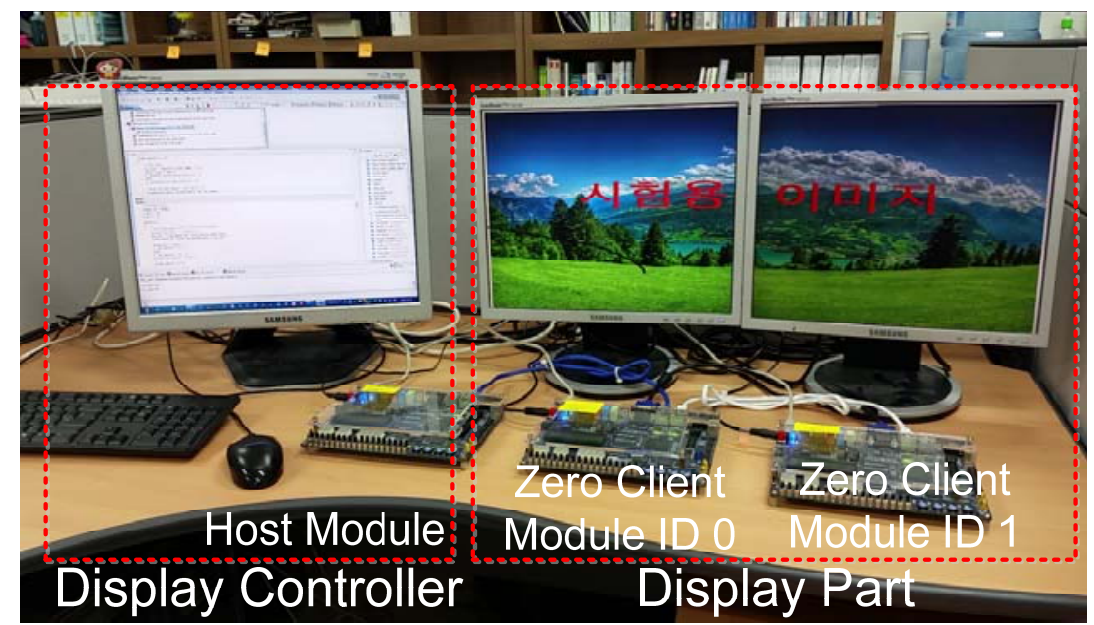

Figure 5. Photograph of our experimental environment

\section{CONCLUSION}

In this paper, we presented our LFD system which consists of the zero client module and the monitors. The host module which is on the display controller side, splits image and transmits UDP packet through Gigabit-Ethernet. The display part consists of multiple zero client modules and each of them connected in Daisy-Chain. These zero client module has a unique module ID for classification of the module. 
Each zero client module controls the monitor for the image display using the received UDP packet from the host module.

The experimental result demonstrates the feasibility of our proposal to the LFD system. The implemented system has an expandability thanks to the connection topology, and it can constitute the more large display system. Although the display controller processes the $640 \times 480$ image because the computation power of zero client system is restricted, this resolution could be improved according to the processing power of the processor. On the display part, the SRAM image buffer is inadequate for a high resolution image because the size. Therefore, this image buffer can be replaced with a higher capacity memory such as DDR memory. It is helpful for the high resolution image display on each zero client module. In the future work, we plan to improve the performance of the zero client system and optimize the UDP packet to implement a more efficient system.

\section{ACKNOWLEDGEMENTS}

This study was supported by the IT R\&D program of MSIP/IITP [10047088, Development of open type Hadoop storage appliance to support more than 48 TB per single data node].

\section{REFERENCES}

[1] Rudolfs Bundulis, et.al., “Concept of Virtual Machine based High Resolution Display Wall”, Information, Electronic and Electrical Engineering, pp. 1-6, Nov 2014.

[2] Kwang-Yong Kim, et.al., "The Design of Server Virtualization Based Video-wall Control System”, International Conference on Computing and Convergence Technology, pp. 401-404, Dec 2012.

[3] Subash K, et.al., "Design and development of a Giga-bit Ethernet based high speed broadband data acquisition system for an underwater imaging array”, OCEANS 2010, pp. 1-7, Sept. 2010.

[4] Sang Don Kim, et.al., "Multi-Energy X-Ray Imaging System Using Single Photon Counting”, International Review on Computers and Software, vol. 8, no. 7, July, 2013.

[5] Samsung, Zero \& Thin Clients, http://www.samsung.com/us/business/computing/zero-clients-and-thin-clients.

[6] Teradici, Simplify and Save with PCoIP Zero Clients: Down Size the End Point, http://www.teradici.com/productsand-solutions/pcoip-products/zero-clients

[7] LG, LG Zero Client \& Teradici PcoIP, http://www.lg.com/us/ commercial/desktop-virtualization

[8] Dell, Cloud Client-Computing and Desktop Virtualization, http://www.dell.com/us/business/p/cloud-connect/pd

[9] Sang Don Kim, et.al., "Architecture for A Zero Client Based Scalable Large Format Display System," International Journal of Computer and Information Technology, 2015.

[10] Altera, DE2-115 Development and Education Board, http://www.altera.com/education/univ/materials/boards/de2115/unv-de2-115-board.html

\section{BIOGRAPHIES OF AUTHORS}

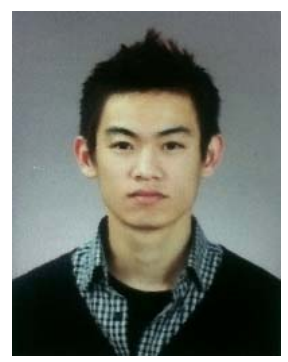

Sang Don Kim is M.S. student in the Department of Electronic Engineering at Seoul National University of Science and Technology, Seoul, Korea. He has a B.S. degree in Electronic Engineering from Seoul National University of Science and Technology, Seoul, Korea. His research interest includes computer architecture, low power circuit design and signal processing.

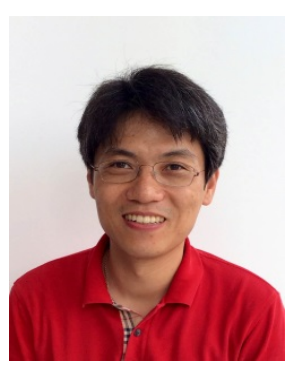

Seung Eun Lee is an Assistant Professor in the Dept. of Electronic Engineering at Seoul National University of Science and Technology. He has a Ph.D. degree in Electrical and Computer Engineering from the University of California, Irvine. His current research interests include computer architecture, multi-processor system-on-chip, low-power and resilient VLSI, and hardware acceleration for emerging applications. Prior to joining Seoul Tech in 2010, he spent 2 years as a platform architect at Intel Labs, Hillosboro, OR, USA and 6 years as a researcher at Korea Electronics Technology Institute, Korea. 